UCRHEP-T566

May 2016

\title{
Gauge $B-L$ Model of Radiative Neutrino Mass with Multipartite Dark Matter
}

\author{
Ernest Ma, Nicholas Pollard, Oleg Popov, and Mohammadreza Zakeri \\ Department of Physics and Astronomy, \\ University of California, Riverside, California 92521, USA
}

\begin{abstract}
We propose an extension of the standard model of quarks and leptons to include gauge $B-L$ symmetry with an exotic array of neutral fermion singlets for anomaly cancellation. With the addition of suitable scalars also transforming under $U(1)_{B-L}$, this becomes a model of radiative seesaw neutrino mass with possible multipartite dark matter. If leptoquark fermions are added, necessarily also transforming under $U(1)_{B-L}$, the diphoton excess at $750 \mathrm{GeV}$, recently observed at the Large Hadron Collider, may also be explained.
\end{abstract}




\section{Introduction :}

It is well-known that a gauge $B-L$ symmetry is supported by a simple extension of the standard model (SM) of quarks and leptons with the addition of one singlet right-handed neutrino per family, so that the theory is anomaly-free. For convenience in notation, let these three extra neutral fermion singlets $N$ be left-handed, then their charges under $U(1)_{B-L}$ are $(1,1,1)$. Their additional contributions to the axial-vector anomaly and the mixed gaugegravitational anomaly are respectively

$$
(1)^{3}+(1)^{3}+(1)^{3}=3, \quad(1)+(1)+(1)=3,
$$

which cancel exactly those of the SM quarks and leptons. On the other hand, it has been known for some time [1] that another set of charges are possible, i.e.

$$
(-5)^{3}+(4)^{3}+(4)^{3}=3, \quad(-5)+(4)+(4)=3 .
$$

Adding also three pairs of neutral singlet fermions with charges $(1,-1)$, naturally small seesaw Dirac masses for the known three neutrinos may be obtained [2], and a residual global $U(1)$ symmetry is maintained as lepton number. A further extension in the scalar sector allows for the unusual case of $Z_{3}$ lepton number [3] with the appearance of a scalar dark-matter candidate which is unstable but long-lived and decays to two antineutrinos. Here we consider another set of possible charges for the neutral fermion singlets, such that tree-level neutrino masses are forbidden. New scalar particles transforming under $U(1)_{B-L}$ are then added to generate one-loop Majorana neutrino masses. The breaking of $B-L$ to $Z_{2}$ results in lepton parity and thus $R$ parity or dark parity [4] which is odd for some particles, the lightest neutral one being dark matter. A closer look st the neutral fermion singlets shows that one may be a keV sterile neutrino, and two others are heavy and stable, thus realizing the interesting scenario of multipartite dark matter. If color-triplet fermions with both $B$ and $L$ are added, the diphoton excess [5, 6] at $750 \mathrm{GeV}$, recently observed at the Large Hadron Collider (LHC), may also be explained. 


\section{Model :}

The extra left-handed neutral singlet fermions have charges $(2,2,2,2,-1,-1,-3)$, so that

$$
4(2)^{3}+2(-1)^{3}+(-3)^{3}=3, \quad 4(2)+2(-1)+(-3)=3 .
$$

Since there is no charge +1 in the above, there is no connection between them and the doublet neutrinos $\nu$ with charge -1 through the one Higgs doublet $\Phi$ which has charge zero. Neutrinos are thus massless at tree level. To generate one-loop Majorana masses, the basic mechanism of Ref. [7] is adopted, using the four fermions with charge +2 , but because of the $U(1)_{B-L}$ gauge symmetry, we need both a scalar doublet $\left(\eta^{+}, \eta^{0}\right)$ and a scalar singlet $\chi^{0}$. The $U(1)_{B-L}$ gauge symmetry itself is broken by $\rho_{2}^{0}$ with charge -2 and by $\rho_{4}^{0}$ with charge

Table 1: Particle content of proposed model.

\begin{tabular}{|c|c|c|c|c|c|c|c|c|}
\hline Particle & $S U(3)_{C}$ & $S U(2)_{L}$ & $U(1)_{Y}$ & $B$ & $L$ & $B-L$ & copies & $R$ parity \\
\hline$Q=(u, d)$ & 3 & 2 & $1 / 6$ & $1 / 3$ & 0 & $1 / 3$ & 3 & + \\
$u^{c}$ & $3^{*}$ & 1 & $-2 / 3$ & $-1 / 3$ & 0 & $-1 / 3$ & 3 & + \\
$d^{c}$ & $3^{*}$ & 1 & $1 / 3$ & $-1 / 3$ & 0 & $-1 / 3$ & 3 & + \\
\hline$L=(\nu, e)$ & 1 & 2 & $-1 / 2$ & 0 & 1 & -1 & 3 & + \\
$e^{c}$ & 1 & 1 & 1 & 0 & -1 & 1 & 3 & + \\
\hline$N$ & 1 & 1 & 0 & 0 & -2 & 2 & 4 & - \\
$S$ & 1 & 1 & 0 & 0 & 1 & -1 & 2 & + \\
$S^{\prime}$ & 1 & 1 & 0 & 0 & 3 & -3 & 1 & + \\
\hline$\Phi=\left(\phi^{+}, \phi^{0}\right)$ & 1 & 2 & $1 / 2$ & 0 & 0 & 0 & 1 & + \\
$\eta=\left(\eta^{+}, \eta^{0}\right)$ & 1 & 2 & $1 / 2$ & 0 & 1 & -1 & 1 & - \\
$\chi^{0}$ & 1 & 1 & 0 & 0 & 1 & -1 & 1 & - \\
$\rho_{2}^{0}$ & 1 & 1 & 0 & 0 & 2 & -2 & 1 & + \\
$\rho_{4}^{0}$ & 1 & 1 & 0 & 0 & 4 & -4 & 1 & + \\
\hline$D_{1}$ & 3 & 1 & $-1 / 3$ & $1 / 3$ & 1 & $-2 / 3$ & 1 & - \\
$D_{2}$ & 3 & 1 & $-1 / 3$ & $1 / 3$ & -1 & $4 / 3$ & 1 & - \\
$D_{1}^{c}$ & $3^{*}$ & 1 & $1 / 3$ & $-1 / 3$ & -1 & $2 / 3$ & 1 & - \\
$D_{2}^{c}$ & $3^{*}$ & 1 & $1 / 3$ & $-1 / 3$ & 1 & $-4 / 3$ & 1 & - \\
\hline
\end{tabular}


-4. The leptoquark fermions $D_{1,2}$ and $D_{1,2}^{c}$ are not necessary for neutrino mass, but are natural extensions of this model if the diphoton excess at $750 \mathrm{GeV}$ requires an explanation. The complete particle content of this model is shown in Table 1.

\section{Radiative Neutrino Mass:}

Using the four $N$ 's, radiative Majorana masses for the three $\nu$ 's are generated as shown in

Fig. 1. Note that $N, \eta, \chi$ all have odd $R$ parity, so that the lightest neutral particle among

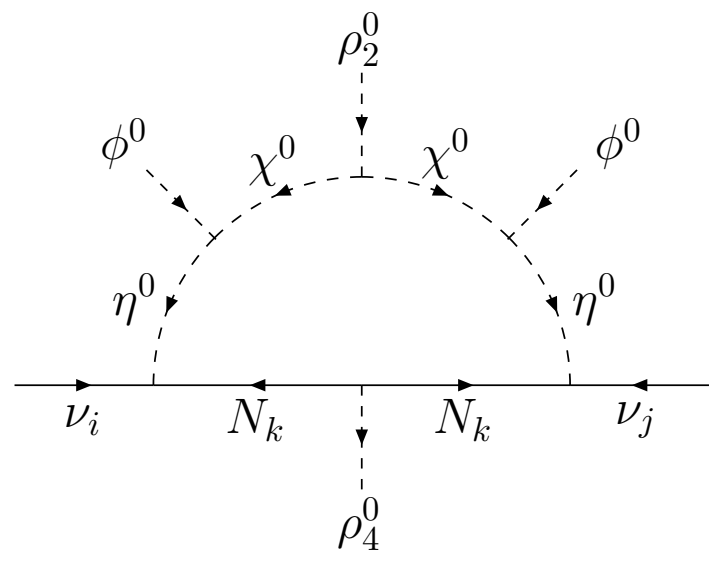

Figure 1: Radiative generation of neutrino mass through dark matter.

them is a dark-matter candidate. This is the scotogenic mechanism, from the Greek 'scotos' meaning darkness. In addition to the $\eta^{\dagger} \Phi \chi$ trilinear coupling used in Fig. 1, there is also the $\eta^{\dagger} \Phi \chi^{\dagger} \rho_{2}$ quadrilinear coupling, which may also be used to complete the loop. There are 4 real scalar fields, spanning $\sqrt{2} R e\left(\eta^{0}\right), \sqrt{2} \operatorname{Im}\left(\eta^{0}\right), \sqrt{2} R e\left(\chi^{0}\right), \sqrt{2} \operatorname{Im}\left(\chi^{0}\right)$. We denote their mass eigenstates as $\zeta_{l}^{0}$ with mass $m_{l}$. Let the $\nu_{i} N_{k} \eta^{0}$ coupling be $h_{i k}^{\nu}$, then the radiative neutrino mass matrix is given by [7]

$$
\left(\mathcal{M}_{\nu}\right)_{i j}=\sum_{k} \frac{h_{i k}^{\nu} h_{j k}^{\nu} M_{k}}{16 \pi^{2}} \sum_{l}\left[\left(y_{l}^{R}\right)^{2} F\left(x_{l k}\right)-\left(y_{l}^{I}\right)^{2} F\left(x_{l k}\right)\right],
$$

where $\sqrt{2} \operatorname{Re}\left(\eta^{0}\right)=\sum_{l} y_{l}^{R} \zeta_{l}^{0}, \sqrt{2} \operatorname{Im}\left(\eta^{0}\right)=\sum_{l} y_{l}^{I} \zeta_{l}^{0}$, with $\sum_{l}\left(y_{l}^{R}\right)^{2}=\sum_{l}\left(y_{l}^{I}\right)^{2}=1, x_{l k}=$ $m_{l}^{2} / M_{k}^{2}$, and the function $F$ is given by

$$
F(x)=\frac{x \ln x}{x-1} .
$$




\section{Multipartite Dark Matter:}

Since the only neutral particles of odd $R$ parity are $N, \eta^{0}, \chi^{0}$, there appears to be only one dark-matter candidate. However as shown below, there could be two or even four, all within the context of the existing model.

First note that $\rho_{2,4}^{0}$ have exactly the right $U(1)_{B-L}$ charges to make the $\left(S, S, S^{\prime}\right)$ fermions massive. The corresponding $3 \times 3$ mass matrix is of the form

$$
\mathcal{M}_{S}=\left(\begin{array}{ccc}
m_{S 1} & 0 & m_{13} \\
0 & m_{S 2} & m_{23} \\
m_{13} & m_{23} & 0
\end{array}\right),
$$

where $m_{S 1}, m_{S 2}$ come from $\left\langle\rho_{2}^{0}\right\rangle=u_{2}$ and $m_{13}, m_{23}$ from $\left\langle\rho_{4}^{0}\right\rangle=u_{4}$. If all these entries are of order $100 \mathrm{GeV}$ to a few $\mathrm{TeV}$, then there are three extra heavy singlet neutrinos in this model which also have even $R$ parity. They do not mix with the light active neutrinos $\nu$ at tree level, but do so in one loop. For example, $S^{\prime}$ mixes with $\nu$ as shown in Fig. 2. Similarly $S$



Figure 2: Radiative generation of $\nu-S^{\prime}$ mixing.

will also mix with $\nu$, using the $S N \chi^{0}$ Yukawa coupling. However, these terms are negligible compared to the assumed large masses for $\left(S, S, S^{\prime}\right)$ and may be safely ignored.

Consider now the possibility that $m_{13}, m_{23}<<m_{S 1}, m_{S 2}$ in $\mathcal{M}_{S}$, then $S^{\prime}$ obtains a small seesaw mass given by

$$
m_{S^{\prime}} \simeq-\frac{m_{13}^{2}}{m_{S 1}}-\frac{m_{23}^{2}}{m_{S 2}} .
$$


Let this be a few $\mathrm{keV}$, then $S^{\prime}$ is a light sterile neutrino which mixes with $\nu$ only slightly through Fig. 2. Hence it is a candidate for warm dark matter. Whereas the usual sterile neutrino is an ad hoc invention, it has a natural place here in terms of its mass as well as its suppressed mixing with the active neutrinos.

We now have the interesting scenario where part of the dark matter of the Universe is cold, and the other is warm. This hybrid case was recently also obtained in a different radiative model of neutrino masses [8]. Within the present context, there is a third possibility. If we assign an extra $Z_{2}$ symmetry, under which $S_{1,2}$ are odd and all other particles even, then the only interactions involving $S_{1,2}$ come from their diagonal $U(1)_{B-L}$ gauge couplings and the diagonal Yukawa terms $f_{1} S_{1} S_{1}\left(\rho_{2}^{0}\right)^{*}$ and $f_{2} S_{2} S_{2}\left(\rho_{2}^{0}\right)^{*}$. This means that both $S_{1}$ and $S_{2}$ are stable and their relic abundances are determined by their annihilation cross sections to SM particles. In this scenario, dark matter has four components 9 .

Since $S_{1,2}$ are now separated from $S^{\prime}$, the $m_{13}$ and $m_{23}$ terms in $\mathcal{M}_{S}$ are zero and there is no tree-level mass for $S^{\prime}$. However, there is a one-loop mass as shown in Fig. 3. This makes

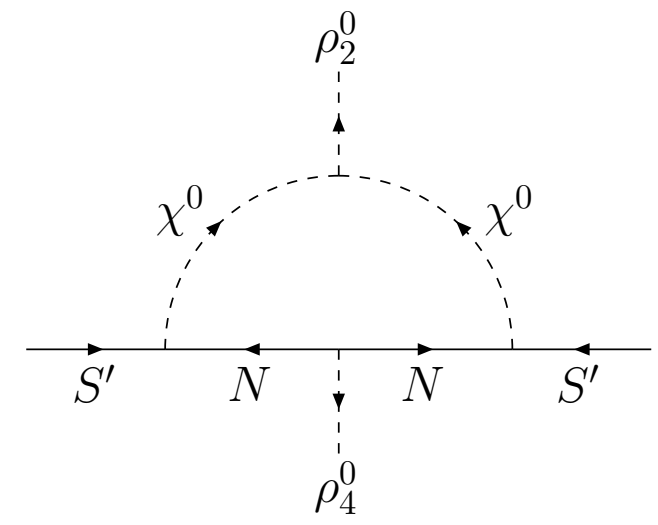

Figure 3: Radiative generation of $S^{\prime}$ mass.

it more natural for $S^{\prime}$ to be light. A detailed study of the dark-matter phenomenology of this multipartite scenario will be given elsewhere. 
Scalar Sector for Symmetry Breaking :

In this model, there is only one Higgs doublet $\Phi$ which breaks the $S U(2)_{L} \times U(1)_{Y}$ electroweak symmetry, whereas there are two Higgs singlets $\rho_{2}$ and $\rho_{4}$ which break $U(1)_{B-L}$ to $Z_{2}$. The most general Higgs potential consisting of $\Phi, \rho_{2}, \rho_{4}$ is given by

$$
\begin{aligned}
V & =\mu_{0}^{2} \Phi^{\dagger} \Phi+\mu_{2}^{2} \rho_{2}^{*} \rho_{2}+\mu_{4}^{2} \rho_{4}^{*} \rho_{4}+\frac{1}{2} \mu_{24}\left[\rho_{2}^{2} \rho_{4}^{*}+H . c .\right]+\frac{1}{2} \lambda_{0}\left(\Phi^{\dagger} \Phi\right)^{2}+\frac{1}{2} \lambda_{2}\left(\rho_{2}^{*} \rho_{2}\right)^{2} \\
& +\frac{1}{2} \lambda_{4}\left(\rho_{4}^{*} \rho_{4}\right)^{2}+\lambda_{02}\left(\Phi^{\dagger} \Phi\right)\left(\rho_{2}^{*} \rho_{2}\right)+\lambda_{04}\left(\Phi^{\dagger} \Phi\right)\left(\rho_{4}^{*} \rho_{4}\right)+\lambda_{24}\left(\rho_{2}^{*} \rho_{2}\right)\left(\rho_{4}^{*} \rho_{4}\right) .
\end{aligned}
$$

Let $\left\langle\phi^{0}\right\rangle=v,\left\langle\rho_{2}\right\rangle=u_{2},\left\langle\rho_{4}\right\rangle=u_{4}$, then the minimum of $V$ is determined by

$$
\begin{aligned}
0 & =\mu_{0}^{2}+\lambda_{0} v^{2}+\lambda_{02} u_{2}^{2}+\lambda_{04} u_{4}^{2}, \\
0 & =\mu_{2}^{2}+\lambda_{02} v^{2}+\lambda_{2} u_{2}^{2}+\lambda_{24} u_{4}^{2}+\mu_{24} u_{4}, \\
0 & =u_{4}\left(\mu_{4}^{2}+\lambda_{04} v^{2}+\lambda_{24} u_{2}^{2}+\lambda_{4} u_{4}^{2}\right)+\frac{1}{2} \mu_{24} u_{2}^{2} .
\end{aligned}
$$

The would-be Goldstone bosons are $\phi^{ \pm}, \sqrt{2} \operatorname{Im}\left(\phi^{0}\right)$, corresponding to the breaking of $S U(2)_{L} \times$ $U(1)_{Y}$ to $U(1)_{e m}$, and $\sqrt{2}\left[u_{2} \operatorname{Im}\left(\rho_{2}\right)+2 u_{4} \operatorname{Im}\left(\rho_{4}\right)\right] / \sqrt{u_{2}^{2}+4 u_{4}^{2}}$, corresponding to the breaking of $U(1)_{B-L}$ to $Z_{2}$. The linear combination orthogonal to the latter is a physical pseudoscalar $A$, with a mass given by

$$
m_{A}=\frac{-\mu_{24}\left(u_{2}^{2}+4 u_{4}^{2}\right)}{2 u_{4}} .
$$

The $3 \times 3$ mass-squared matrix of the physical scalars $\left[\sqrt{2} R e\left(\phi^{0}\right), \sqrt{2} R e\left(\rho_{2}\right), \sqrt{2} R e\left(\rho_{4}\right)\right]$ is given by

$$
\mathcal{M}^{2}=\left(\begin{array}{ccc}
2 \lambda_{0} v^{2} & 2 \lambda_{02} v u_{2} & 2 \lambda_{04} v u_{4} \\
2 \lambda_{02} v u_{2} & 2 \lambda_{2} u_{2}^{2} & u_{2}\left(2 \lambda_{24} u_{4}+\mu_{24}\right) \\
2 \lambda_{04} v u_{4} & u_{2}\left(2 \lambda_{24} u_{4}+\mu_{24}\right) & 2 \lambda_{4} u_{4}^{2}-\mu_{24} u_{2}^{2} / 2 u_{4}
\end{array}\right) .
$$

For $v^{2}<<u_{2,4}^{2}, \sqrt{2} \operatorname{Re}\left(\phi^{0}\right)=h$ is approximately a mass eigenstate which is identified with the $125 \mathrm{GeV}$ particle discovered at the LHC. 


\section{Gauge Sector:}

Since $\phi^{0}$ does not transform under $U(1)_{B-L}$ and $\rho_{2,4}$ do not transform under $S U(2)_{L} \times U(1)_{Y}$, there is no tree-level mixing between their corresponding gauge bosons $Z$ and $Z_{B-L}$. In our convention, $M_{Z_{B-L}}^{2}=8 g_{B-L}^{2}\left(u_{2}^{2}+4 u_{4}^{2}\right)$. The LHC bound on $M_{Z_{B-L}}$ comes from the production of $Z_{B-L}$ from $u$ and $d$ quarks and its subsequent decay to $e^{-} e^{+}$and $\mu^{-} \mu^{+}$. If all the particles listed in Table 1 are possible decay products of $Z_{B-L}$ with negligible kinematic suppression, then its branching fraction to $e^{-} e^{+}$and $\mu^{-} \mu^{+}$is about 0.061 . The $c_{u, d}$ coefficients used in the LHC analysis [10, 11] are then

$$
c_{u}=c_{d}=\left[\left(\frac{1}{3}\right)^{2}+\left(\frac{1}{3}\right)^{2}\right] g_{B-L}^{2} \times B\left(Z_{B-L} \rightarrow e^{-} e^{+}, \mu^{-} \mu^{+}\right)=1.36 \times 10^{-2} g_{B-L}^{2} .
$$

From LHC data based on the 7 and $8 \mathrm{TeV}$ runs, a bound of about $2.5 \mathrm{TeV}$ would correspond to $g_{B-L}<0.24$.

\section{Leptoquark Fermions:}

The singlet leptoquark fermions $D_{1,2}$ have charge $-1 / 3$ and the following possible interactions:

$$
D_{1} d^{c} \chi^{*}, \quad D_{2} d^{c} \chi, \quad D_{1} D_{2}^{c} \rho_{2}^{*}, \quad D_{2} D_{1}^{c} \rho_{2}
$$

Hence they mix in a $2 \times 2$ mass matrix linking $D_{1,2}$ to $D_{1,2}^{c}$ with $\left\langle\rho_{2}\right\rangle=u_{2}$, and decay to $d$ quarks $+\chi\left(\chi^{*}\right)$. Now $\chi$ mixes with $\eta^{0}$, so it decays to neutrinos $(\nu)$ and dark matter $(N)$, which are invisible. The search for $D_{1,2}$ at the LHC would be similar to the search for scalar quarks which decay to quarks + missing energy. However, if we assume that $N$ has a mass of about $200 \mathrm{GeV}$, then there is no useful limit at present on the mass of $D_{1,2}$ from the LHC.

Consider now the pseudoscalar $A$ of Eq. (12). Let the two mass eigenstates in the $\left(D_{1,2}, D_{1,2}^{c}\right)$ sector be $\psi_{1,2}$, then $A$ couples to them according to

$$
\mathcal{L}_{\text {int }}=f_{1} \bar{\psi}_{1} \gamma_{5} \psi_{1}+f_{2} \bar{\psi}_{2} \gamma_{5} \psi_{2}
$$

where $f_{1,2}$ are rearranged from their original $D_{1} D_{2}^{c} \rho_{2}^{*}$ and $D_{2} D_{1}^{c} \rho_{2}$ couplings. Hence $A$ decays 
to two gluons as well as to two photons in one loop through $\psi_{1,2}$. It may also decay to dark matter, say $N N$, at tree level. It is thus a possible candidate for explaining the $750 \mathrm{GeV}$ diphoton excess recently observed [5, 6] at the LHC. The numerical analysis of this model runs parallel to that of a recent proposal [12], and will not be repeated here. Note again that these leptoquark fermions are not essential for the radiative generation of neutrino masses based on $B-L$.

\section{Conclusion :}

Using gauge $U(1)_{B-L}$ symmetry, we have proposed a new anomaly-free solution with exotic fermion singlets, such that neutrino mass is forbidden at tree level. We add a number of new scalars so that neutrino masses are obtained in one loop through dark matter, i.e. the scotogenic mechanism. Because of the structure of the new singlets required for anomaly cancellation, we find a possible dark-matter scenario with four components. Three are stable cold Weakly Interaction Massive Particles (WIMPs) and one a keV singlet neutrino, i.e. warm dark matter with a very long lifetime. If leptoquark fermions are added, transforming under $U(1)_{B-L}$, the recently observed $750 \mathrm{GeV}$ diphoton excess may also be explained.

Acknowledgement : This work was supported in part by the U. S. Department of Energy Grant No. de-sc0008541.

\section{References}

[1] J. C. Montero and V. Pleitez, Phys. Lett. B675, 64 (2009).

[2] E. Ma and R. Srivastava, Phys. Lett. B741, 217 (2015).

[3] E. Ma, N. Pollard, R. Srivastava, and M. Zakeri, Phys. Lett. B750, 135 (2015).

[4] E. Ma, Phys. Rev. Lett. 115, 011801 (2015). 
[5] The ATLAS Collaboration, ATLAS-CONF-2015-81.

[6] The CMS Collaboration, CMS-PAS-EXO-15-004.

[7] E. Ma, Phys. Rev. D73, 077301 (2006).

[8] R. Adhikari, D. Borah, and E. Ma, Phys. Lett. B755, 414 (2016).

[9] Q.-H. Cao, E. Ma, J. Wudka, and C.-P. Yuan, arXiv:0711.3887 [hep-ph].

[10] G. Aad et al. (ATLAS Collaboration), Phys. Rev. D90, 052005 (2014).

[11] S. Khachatryan et al. (CMS Collaboration), JHEP 1504, 025 (2015).

[12] S. Fraser, C. Kownacki, E. Ma, N. Pollard, O. Popov, and M. Zakeri, arXiv:1603.04778 [hep-ph]. 